\title{
DDS-Suite - A Dynamic Data Acquisition, Processing, and Analysis System for Wind Tunnel Testing
}

\author{
Nathan J. Burnside ${ }^{*}$ \\ NASA Ames Research Center, Moffett Field, CA 94035
}

\begin{abstract}
Wind Tunnels have optimized their steady-state data systems for acquisition and analysis and even implemented large dynamic-data acquisition systems, however development of near real-time processing and analysis tools for dynamic-data have lagged. DDS-Suite is a set of tools used to acquire, process, and analyze large amounts of dynamic data. Each phase of the testing process: acquisition, processing, and analysis are handled by separate components so that bottlenecks in one phase of the process do not affect the other, leading to a robust system. DDS-Suite is capable of acquiring 672 channels of dynamic data at rate of $275 \mathrm{MB}$ / s More than 300 channels of the system use 24-bit analog-to-digital cards and are capable of producing data with less than $0.01^{\circ}$ of phase difference at $1 \mathrm{kHz}$. System architecture, design philosophy, and examples of use during NASA Constellation and Fundamental Aerodynamic tests are discussed.
\end{abstract}

\section{Introduction}

$\mathrm{R}$ ESEARCHERS at NASA Ames have developed and implemented a suite of dynamic data tools for use in wind tunnels to acquire, process, and analyze large amounts of dynamic data with minimal human input. Use of the new dynamic data system in eight major aeroacoustic tests in the Space Shuttle, Constellation, and Fundamental Aerodynamics programs has proven its reliability and usefulness for high channel-count dynamic-data testing. Fully processed preliminary data are available for analysis and plotting minutes after acquisition, giving researchers the tools to make test matrix or priority changes. The system is modular and scalable allowing for rapid deployment in tests needing only a handful of channels or hundreds of channels. Current hardware allows for approximately 275 MB / s of data acquisition over 672 channels of discretely sampled data. Sample rates of up to $204.8 \mathrm{kHz}$ are sustainable for sample durations of 20 seconds.

This paper will discuss the design philosophy of the 672-channel Dynamic Data System Suite (DDS-Suite) and its applications for dynamic-data testing in the Ames Unitary Wind Tunnels and the Arnold Engineering Development Center (AEDC) 40- by 80-Foot Wind Tunnel. There are three main components of DDS-Suite: (1) $L A M D A$, the acquisition software; (2) ProcessScheduler, the processing portion of the suite; and (3) QueryGUI, the data plotting and analysis tool. Specific application to a number of Constellation and Space Shuttle tests will be discussed highlighting the robustness and adaptability of the DDS-Suite.

\section{Background}

Wind tunnel data acquisition and processing has evolved over the last seven decades with large gains in data accuracy, precision, quantity, and productivity rates. Production wind tunnels like the NASA Ames 11-Foot and 9x7-Foot have focused on optimizing their steady-state data systems like those that acquire and process tunnel conditions, steady surface-pressures, model position information, temperature data, and balance measurements. The Standard Data System (SDS) offers real-time steady-state data viewing, analysis, and plotting and has been in constant use for the last 15 years ${ }^{1}$. Dynamic-data acquisition systems that collect data for accelerometers, microphones, and unsteady surface-pressures however, lagged in the evolutionary process. Closed-loop dynamicdata acquisition, processing, and analysis tools were primitive until the latter half of the last decade. The ability to acquire large amounts of dynamic-data existed, but fast and efficient methods of processing and analysis did not. DDS-Suite automates the acquisition and processing of test data, making it available for plotting minutes after testing.

\footnotetext{
${ }^{*}$ Aerospace Engineer, Experimental Aero-Physics Branch, AIAA Senior Member
}

1

American Institute of Aeronautics and Astronautics 
IS-21, a 3\% Space Shuttle aeroacoustics test supporting the return-to-flight efforts in the wake of the Space Shuttle Columbia accident drove the initial development of DDS-Suite. During test planning it became apparent that existing dynamic-data systems did not have the capacity or processing throughput to meet the test requirements. DDS-Suite was designed to make preliminary acoustic data from 117 model mounted unsteady-pressure sensors available for plotting less than 5 minutes after acquisition ${ }^{2}$. Network communications with SDS created a closedloop dynamic-data triggering process that greatly improved productivity. Automated processing created narrowband and 1/3-octave data that were inserted into a database for instant data availability. The productivity of IS-21 can be compared directly with IS-2, a Space Shuttle acoustic test performed in the 1970's with 120 transducers. Data from IS-2 was recorded on analog tapes and took more than 2 years to reduce and analyze ${ }^{3}$ compared with IS- 21 that used five data analysts to plot and analyze data in near realtime.

\section{DDS-Suite Design}

The global design philosophy for DDS-Suite was to create a robust, scalable, user-friendly software and hardware system that would have minimal impact on normal wind tunnel operations. Three distinct phases are involved in dynamic data measurements: (1) data acquisition; (2) processing; and (3) analysis. Each phase of operation in $D D S$-Suite is independent. Figure 1 shows the major software components of each phase and the common links. ProcQueue is the processing queue and it links the acquisition and processing phases. The ProcessedData database stores results and links processing with analysis. A bottleneck in processing does not affect data acquisition or the availability of existing processed data. Acquisition and processing require minimal user interaction once the system is started providing an enormous throughput of data.

All data phases are equally important for obtaining the

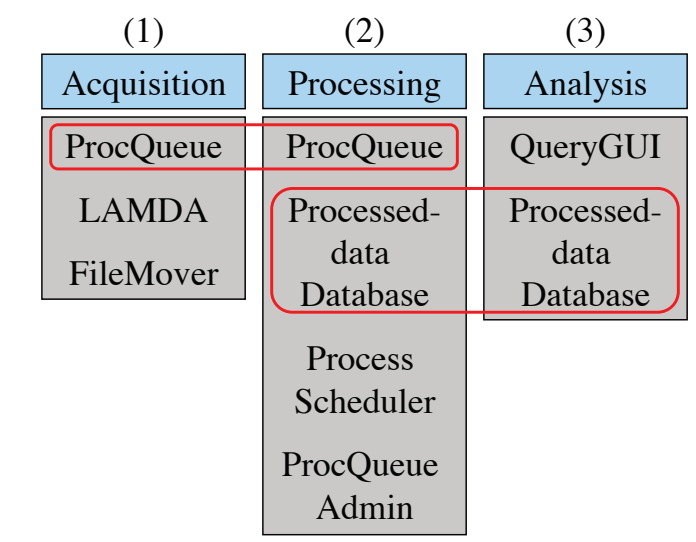

Figure 1. Each phase of DDS-Suite has several software components. final results, but data acquisition carries the most risk because of wind tunnel operation costs. Total wind-on costs, including power can approach $\$ 10,000 /$ hour making data acquisition efficiency paramount. $L A M D A$, the data acquisition program was designed to acquire and store data as quickly as possible while maintaining robustness. Scalability was an important consideration in system design. Response to doubling channel count or the number of data analysts, needed to be as simple as adding twice as many computers. Achieving a scalable architecture in DDS-Suite was accomplished differently for each phase of the data handling and will be discussed below.

\section{A. Acquisition}

LAMDA is the data acquisition portion of DDS-Suite. It was designed to interact with SDS, the wind tunnel static data system or as a stand-alone user driven system. The system consists of at least one master computer and a hardware chassis containing analog-to-digital converters. Slave computers and chassis are added to increase system channels. Communication with motor controllers and other measurement systems such as Pressure Sensitive Paint (PSP) system greatly increases productivity and is coordinated through the master computer.

HP and Agilent 1432 and 1433 data acquisition cards operating in VXI chassis were the development target for LAMDA. The practical limit for the number of 1433 8-channel A/D cards in a VXI chassis is nine because of power limitations. It is possible to span the VXI bus across multiple chassis with MXI-bus extender cards, but all of the data from the extended bus has to flow to the host computer using an IEEE 1394a (Firewire 400) connection. This limitation drove the modular architecture of one computer host for each chassis of data acquisition hardware. By using a master / slave arrangement, computers and chassis are added to meet the channel requirements while maintaining data throughput. The scalable architecture of DDS-Suite allows for test complexity to increase without effecting productivity. Recent improvements allow for combinations of older VXI and newer PXI hardware. Figure 2 shows a typical system deployment for a high-channel count test; note that there is one master computer and 11 slaves.

LAMDA uses Remote Procedure Calls (RPCs) to communicate with SDS, creating a closed-loop data acquisition process that is synced with model movement and wind tunnel conditions. Communication between the master and slave data acquisition computers is accomplished using network TCP packets on the gigabit network (1000 Mb/s). 
The data acquisition chassis are all triggered at the same time using a hardware trigger. All triggering lines are equal lengths to maintain phase, although this is not indicated in Fig. 2.

During a typical pitch-pause sweep the Facility Control System (FCS) stops at each angle-of-attack in the run vector and notifies SDS that the tunnel is on condition. Meanwhile the master computer polls SDS to see if the
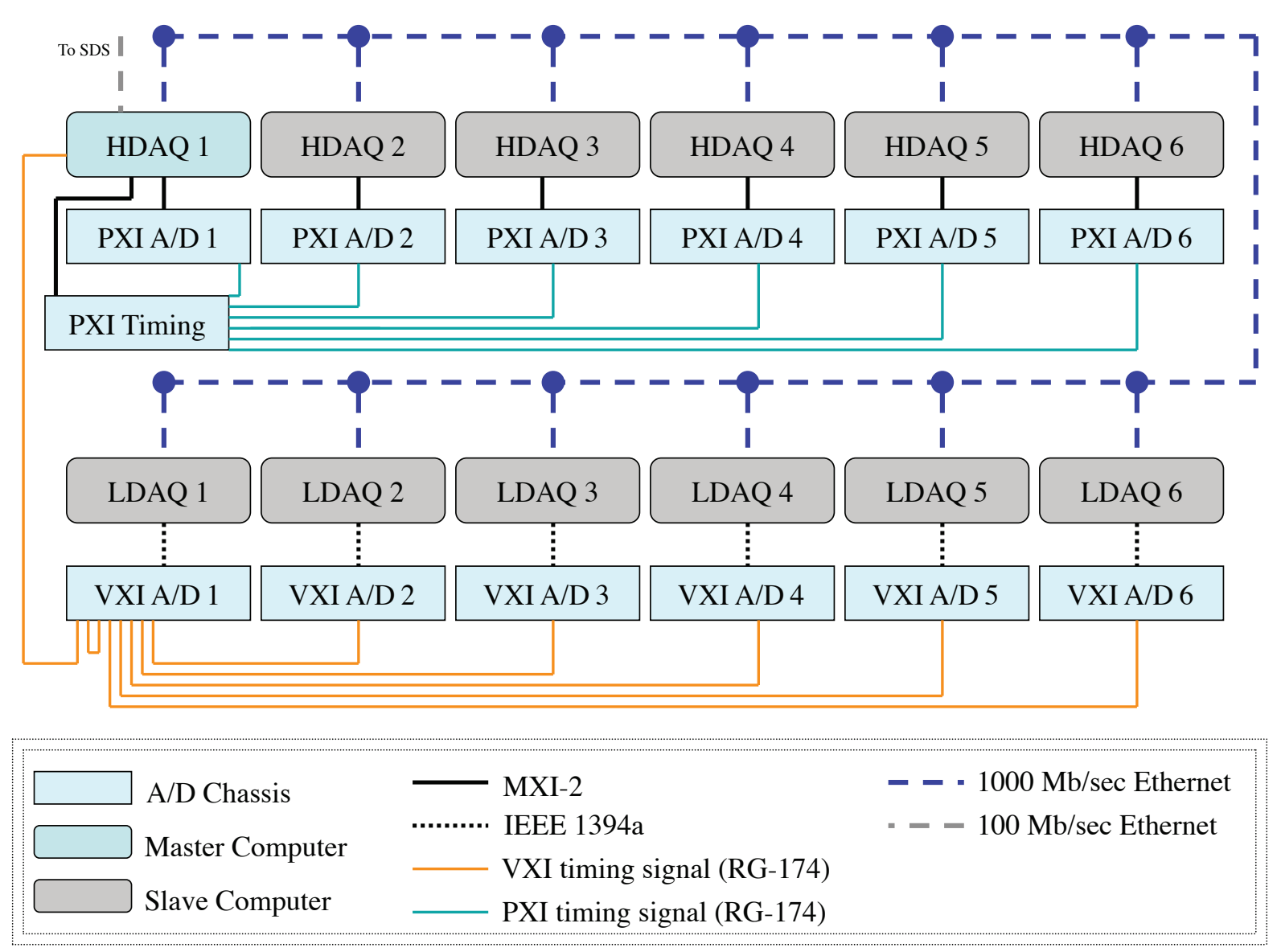

Figure 2. $L A M D A$ system schematic showing a typical 576-channel deployment. Note that the data inputs to the analog-to-digital converters are not shown.

tunnel is on condition. This polling occurs at a rate of $10 \mathrm{~Hz}$. When SDS confirms that the tunnel is on condition, the master commands SDS to take a static data point and then asks SDS the run and sequence number of the data being acquired. The run and sequence information is sent to all of the slaves via the gigabit network. Each slave replies with a positive echo indicating they are ready for dynamic data acquisition. A hardware trigger sent by the master to all of the A/D chassis starts data acquisition. After each slave finishes acquiring its data, it checks back in with the master and then stores the data to a local hard disk in a temporary folder. After storing the data, each acquisition computer inserts information about the last data point into the ProcQueue database. Only after each slave completes data acquisition will the master computer tell SDS that the data point is complete. SDS then tells FCS that the data point is complete and it moves the model or tunnel to the next condition in the run vector. Typical 5-second data points take about 6 seconds from start to finish and the time to move the model varies, but 3 to 4 seconds is common.

FileMover is a software program that runs on each acquisition computer but is independent of LAMDA. As its name implies, FileMover moves files from the temporary directory into a directory structure on the local hard drive and onto a network file server that is organized by test, run, and sequence number. Once two copies of the file are made the file in the temporary directory is deleted and a status flag in the ProcQueue database is changed to indicate the file has been moved to the server and is ready for processing. Duplicate copies of the data are important to reduce the inherent risk of computer or hard drive failure. Decoupling the acquisition program from network file transfers reduces the acquisition cycle time and improves reliability. 
LAMDA was originally designed to use the VXI 1433a, 1433b, and 1432a A/D cards manufactured by HP, Agilent, or VXI Technologies. The 1433 cards are 8-channel 16-bit analog-to-digital converters capable of sampling simultaneously at $196.6 \mathrm{kSamples}$ per second. Input ranges are user selectable; the smallest range is \pm 0.005 volts and the largest is \pm 10 volts. The 1432a cards are 16-channel, 16-bit analog-to-digital converters with a maximum sample rate of $51.2 \mathrm{kSamples}$ per second. Their smallest input range is \pm 0.1 volts and the largest is \pm 20 volts. The VXI hardware performs extremely well, but without complicated inter-chassis connections, simultaneous sampling is limited to one chassis. The practical limit of cards in each chassis is nine, meaning the maximum number of correlated channels is 72 or 144 for the 1433 and 1432 cards, respectively.

Prior to the Crew Exploration Vehicle (CEV) 51-AS test in October of 2008, LAMDA received a major upgrade and expanded capability. LAMDA was modified to control a 300-channel PXI based data system in addition to the existing 300-channel VXI system. The National Instruments (NI) system uses PXI-4462 analog-to-digital converters with 24-bit resolution and samples rates as high as $204.8 \mathrm{kSamples}$ per second. The smallest input range is \pm 0.316 volts and the largest is \pm 42.4 volts. Each data acquisition chassis has a PXI-6652 timing card connected to a master timing chassis with a PXI-6652 timing card for each acquisition chassis as shown in Fig. 2. The dedicated timing chassis allows the sample clock and trigger signals to be distributed to several hundred channels creating a massive high-speed data system with simultaneous sampling across all channels.

Phase performance of the PXI system was evaluated using six chassis with 48 channels each. Testing was performed with a $1 \mathrm{kHz}, 1$ volt sine wave. Phase was measured between several channels and follows the nomenclature chassis-channel. The worst phase performance was between $1-1$ and 3-1 at $-0.0018^{\circ}$ and the best performance was $-0.0003^{\circ}$ between $1-1$ and $4-1$. Table 1 summarizes the phase test results for the PXI system.

\begin{tabular}{|c|c|c|}
\hline Chassis-Chan & Chassis-Chan & Phase (deg) \\
\hline $1-1$ & $1-4$ & -0.0009 \\
\hline $1-1$ & $1-48$ & -0.0015 \\
\hline $1-1$ & $2-1$ & -0.0007 \\
\hline $1-1$ & $3-1$ & -0.0018 \\
\hline $1-1$ & $4-1$ & -0.0003 \\
\hline $1-1$ & $5-1$ & -0.0007 \\
\hline $1-1$ & $6-1$ & -0.0015 \\
\hline
\end{tabular}

Table 1. LAMDA phase measurements using a 1 $\mathrm{kHz}, 1$ volt sine wave.

\section{B. Processing}

The ProcQueue database is the central coordinator for data processing and ensures that each data file is processed only once. It contains test, run, sequence number, file name, location status and processing status, and processing priority information for every file acquired by $D D S$ Suite. The ProcQueue database is updated automatically by several components of $D D S$ Suite, however it can be manipulated manually using ProcQueueAdmin. Radio buttons based on the file status are used to filter the data files displayed and status changes are made by highlighting files and using the status change buttons as shown in Fig. 3 where run 70 sequence 1 is processing, run 70 sequence 2 is waiting to be processed, and run 70 sequence 4 is still on the acquisition computer local disks. Each request to the ProcQueue database for a set of files to process, generates a unique process number, this number is assigned to the processed flag for each file in the set so they are easily identifiable. By default all files have the same priority for processing and the queue operates on a first in first out scheme, however the priority can easily changed using ProcQueueAdmin if desired.

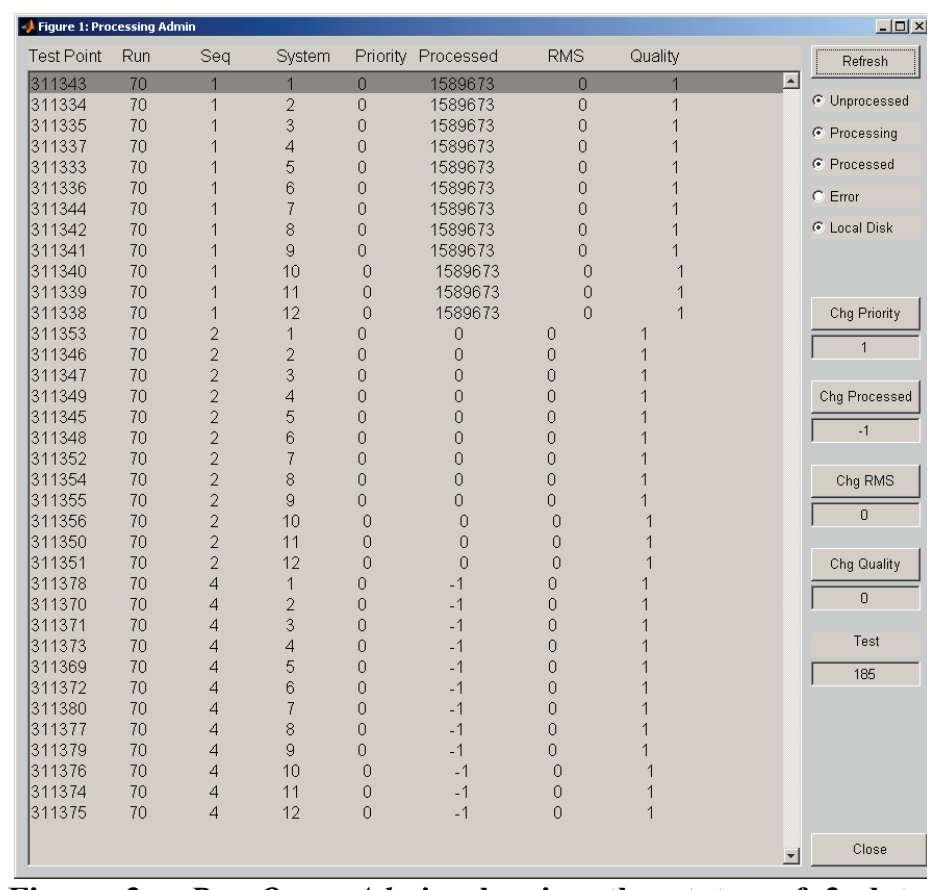

Figure 3. ProcQueueAdmin showing the status of 3 data points. There are 12 files associated with each point.

4

American Institute of Aeronautics and Astronautics 
ProcessScheduler is the software that actually does the processing. Following the modular, scalable architecture any number of independent processing machines can be deployed for a given wind tunnel test. Each instance of ProcessScheduler sends a request to the ProcQueue database asking for the next set of files that need processing. The database returns either a zero indicating there are no files to process or a unique process number. The database software handles request such that files are in use by only one instance of ProcessScheduler at a time. A large test deployment like the one shown in Fig. 2 would have eight computers processing data in near real-time.

ProcesssScheduler is customized for each test but for most applications it processes time history data to create narrowband spectra, 1/3-octave levels, and overall sound pressure levels (OASPL) scaled to full-scale flight levels. The processed data are inserted into a Firebird 1.5.2 database for storage and analysis. The relational database includes information about wind tunnel conditions, model positions, and the processed data. The database structure is capable of supporting multiple tests allowing for data comparison. Figure 4 shows the database schema for the processed data. The primary key(s) for each table are shown in orange. Notice the connections between tables show a one-to-many mapping for the TestDataPoint and channelinfo tables. TestDataCache is a table to store unique values of each data parameter in the TestDataPoint table to improve QueryGUI start times. Entries to the TestDataCache table are transparent to the user and are handled by stored procedures and table triggers.

\section{IS-21 Dynamic Data Results Schema (v.9)}

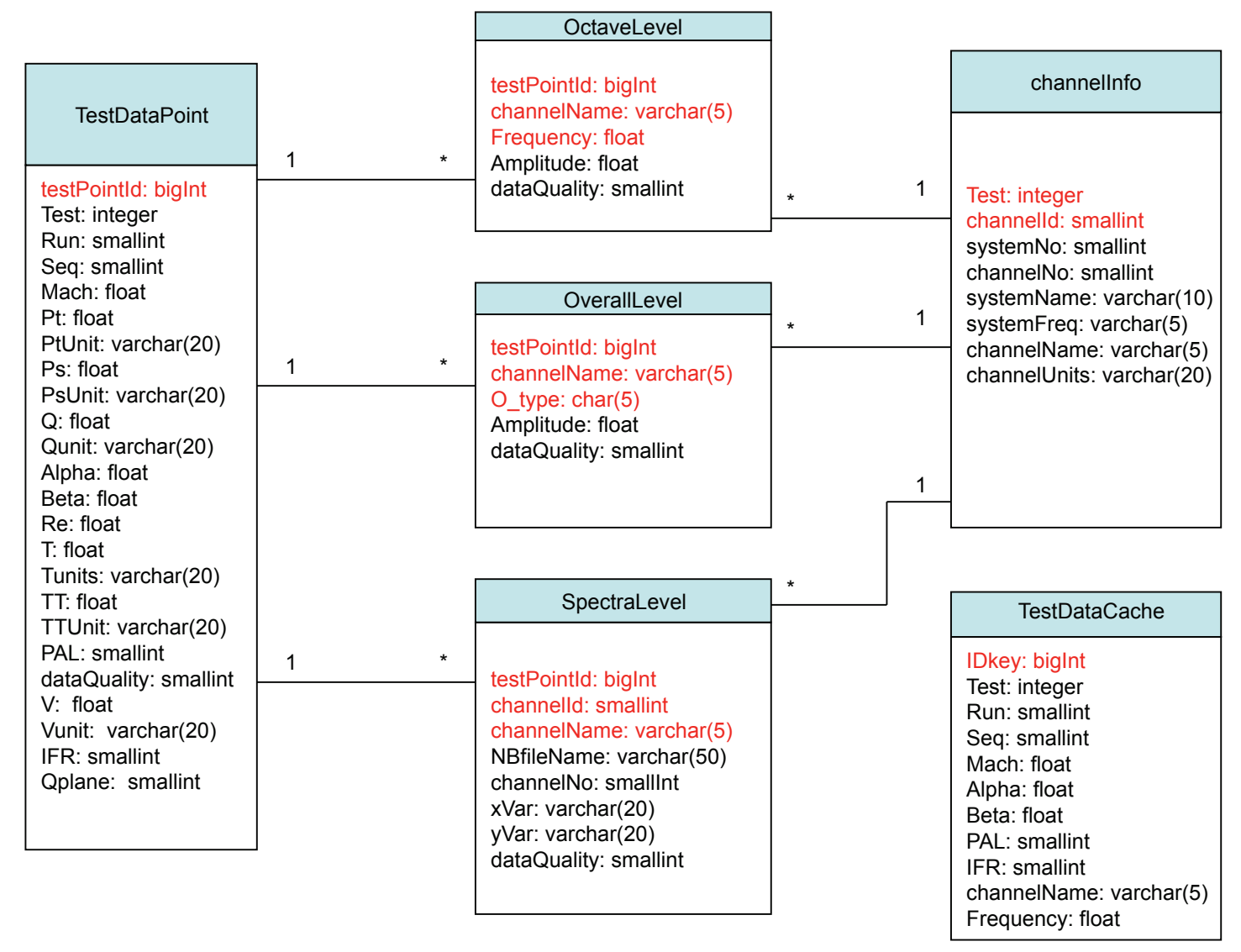

Figure 4. Relational database schema for the ProcessedData database. Primary key(s) for each table are indicated by red variables.

\section{Analysis}

The third component of DDS-Suite is QueryGUI, an analysis and plotting program that allows the user to create database queries using drop down menus. QueryGUI and the ProcessedData database highlight the scalability of DDS-Suite by accommodating user scenarios ranging from a single user with a laptop to dozens of users accessing the data via server. Its simple graphical user interface allows users to create complex database searches. 
Figure 5 shows QueryGUI and several of the parameters available for query. Users make graphical selections of search criteria and then build their query. Graphical selections are converted to a single SQL statement for execution on the ProcessedData database. Once a query is executed results are returned to QueryGUI where the user can view the data or choose from several plotting options. 1/3octave plotting is often the most useful as shown in Fig. 6. Large data sets can also be condensed into a grid plot as shown in Fig. 7, where $\mathrm{x}$-axis of the grid plot is $1 / 3$-octave frequency and the y-axis is sensor channel; color indicates the amplitude. This type of plot is very useful when sensors are distributed evenly over the surface of a vehicle because acoustic hotspots in both frequency and location are identified.

QueryGUI was developed for

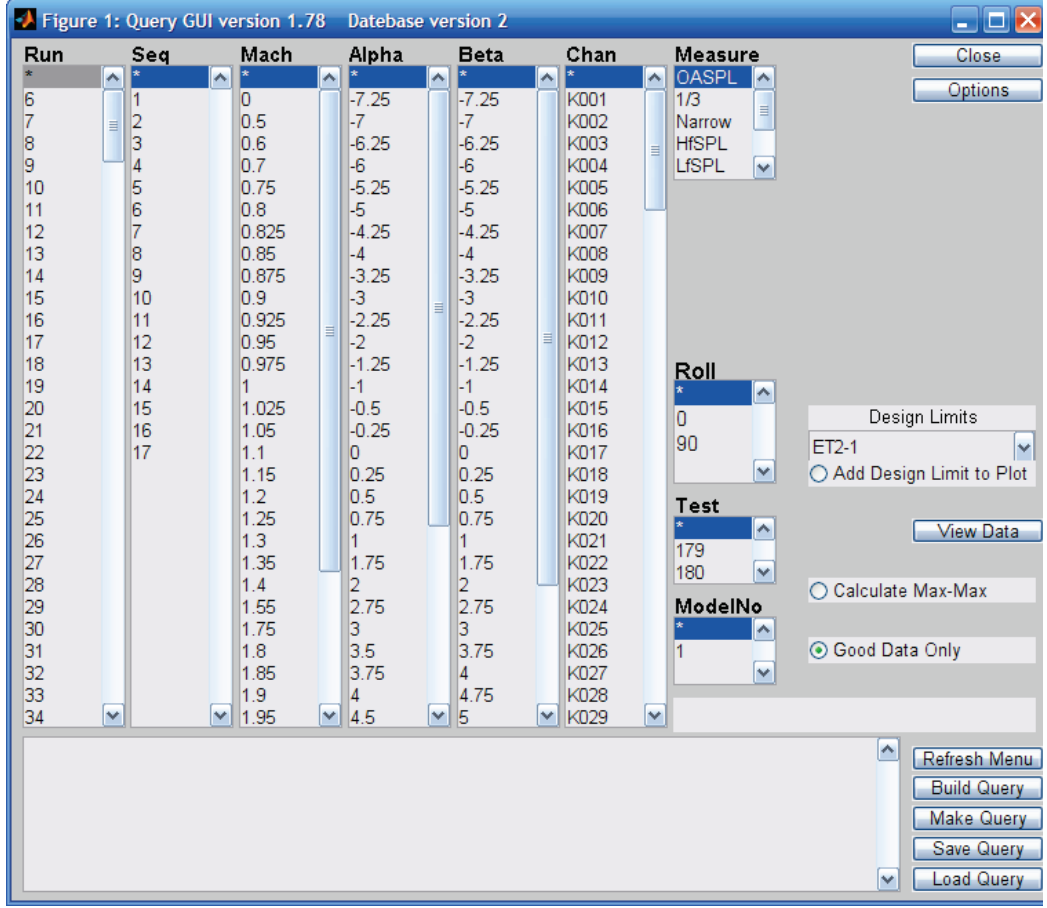

Figure 5. QueryGUI user interface for creating SQL queries to the ProcessedData database.

vehicle aeroacoustic environment testing where zonal acoustic environments are defined by the envelope of the $1 / 3$-octave spectra within the each zone. QueryGUI and the ProcessedData database have a search function called maximax for finding an envelope of all search criteria contained within the query for each transducer and frequency. Because maximax searches often involve millions of database records, the search is performed by a stored procedure in the ProcessedData database. Stored procedures are efficient to execute and avoid memory issues on the host computer because only results of the procedure are returned and not the millions of records that satisfy the search criteria. The maximax stored procedure is built into the database schema and has been optimized to return results in a reasonable time period.

\section{Applications}

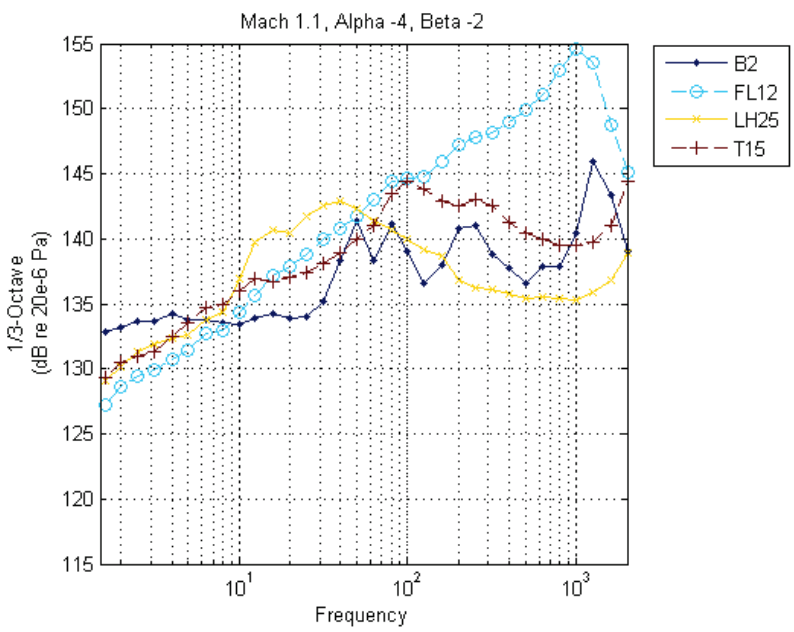

Figure 6. 1/3-Octave results plotted by QueryGUI.

\section{CLV Reentry Test}

The Crew Launch Vehicle (CLV) Reentry test demonstrated the flexibility of DDS-Suite. The CLV Reentry test used a $2.8 \%$ First Stage Model to simulate atmospheric reentry. Reentry conditions can vary greatly making the acoustic environment difficult to measure. Angle-of-attack was varied from $90^{\circ}$ to $180^{\circ}$ and roll varied from $0^{\circ}$ to $180^{\circ}$. Model roll was controlled by $B D A S$, an auxiliary data system capable of motor control. $B D A S$ was commanded through $L A M D A$ to maintain a closed loop data system. 


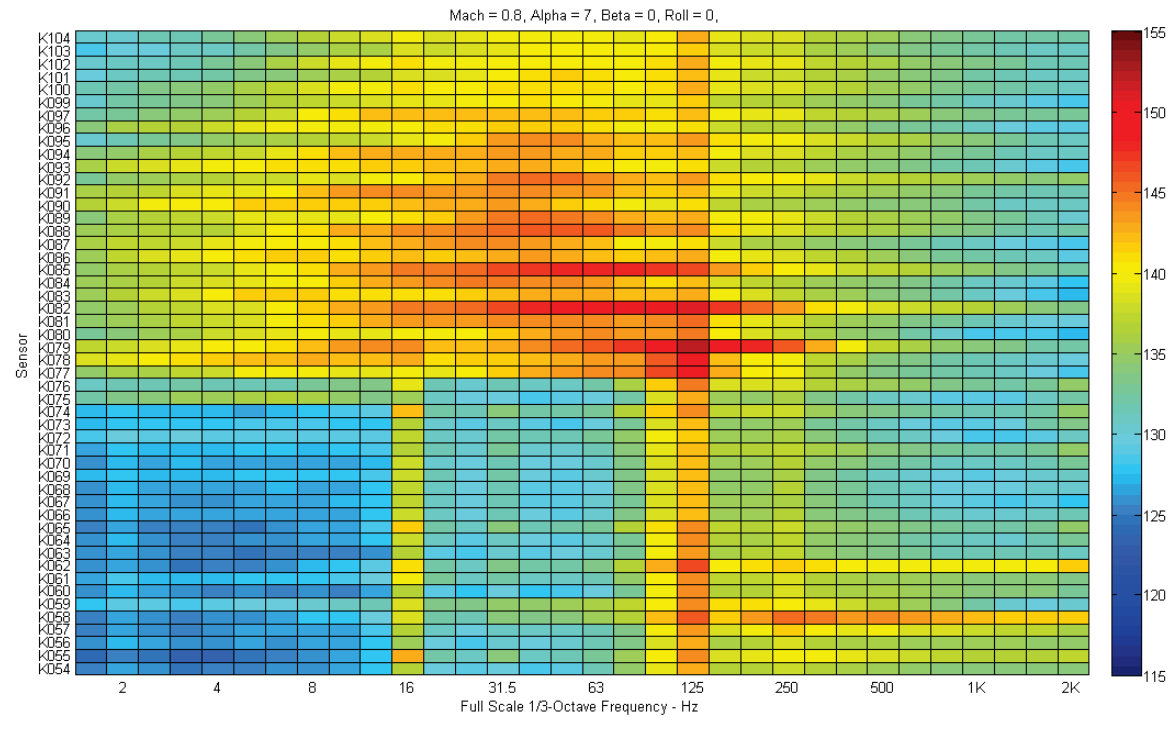

Figure 7. Grid plot produced by QueryGUI shows the amplitudes for 150 sensors over the entire 1/3-octave range.

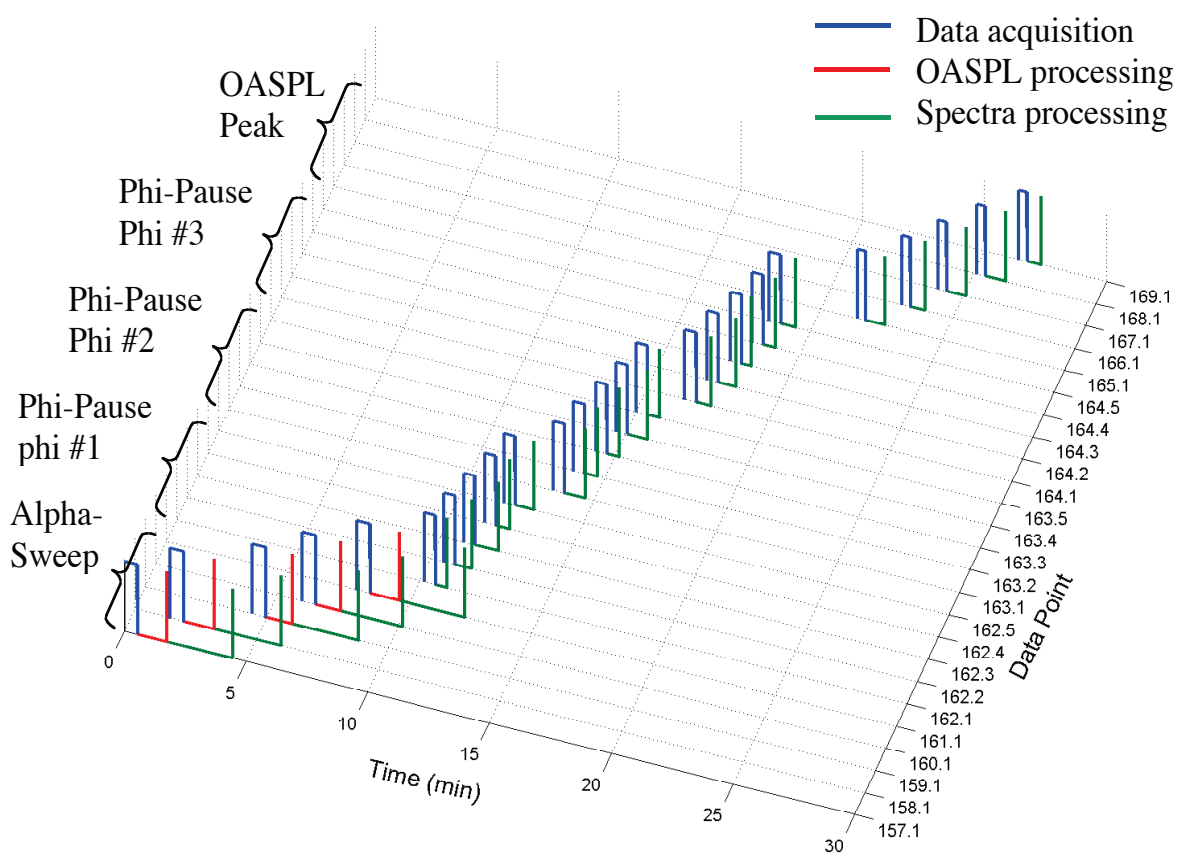

Figure 8. A graphical representation of processing and acquisition time for the CLV Reentry test.

To efficiently measure data over the large ranges of model position, a combination of three data taking modes was used: (1) alpha-sweep; (2) phi-pause; and (3) OASPL peak. During the alpha-sweep mode, data were acquired constantly over a 45 second period while the model was swept through a $30^{\circ}$ range of angle-of-attack. These data were then integrated into OASPL levels with angle-of-attack as the limits of integration. Alpha sweeps were run at five distinct roll angles. The purpose of the alpha sweeps was to identify regions of interest for further integration with fixed angle-of-attack and roll. These fixed data points were the third type of data, OASPL peak. While engineers made decisions about what OASPL peak conditions to test, the phi-pause data were acquired. 
Near real-time decisions about which conditions for the OASPL peak points were made minutes after sweep data were acquired. Approximately 10 minutes separated the end of the alpha sweeps and the start of the OASPL peak data. Figure 8 shows the timing and duration of the acquisition and processing for each of the three data types. This novel data acquisition and processing allowed almost instantaneous analysis of a tremendous amount of data.

\section{E. CEV 80-AS Hot Helium Test}

Another test that demonstrated the flexibility and reliability of DDS-Suite was the CEV 80-AS test. As the single most expensive wind tunnel test in the CEV test program, data system robustness was critical. The test involved temporarily installing a $13 \mathrm{MW}$ heater and its associated piping in the NASA Ames 11-Ft Wind Tunnel to heat 600 psi helium to $700^{\circ} \mathrm{F}$ for simulation of the CEV abort conditions. The operational method of the hot gas delivery system limited each helium test point to 30 seconds and a limited supply of helium allowed for about 20 test points per day making data system reliability more critical than usual.

$L A M D A$ was modified to interact with the helium delivery control system to minimize helium use and to ensure that data were acquired for every helium test point. Test requirements dictated that the helium temperature just before expansion through the four launch abort nozzles be at least $660^{\circ} \mathrm{F}$, however the thermal mass of the piping system damped the temperature response. $L A M D A$ monitored the helium temperature at the model and started data acquisition when it exceeded $660^{\circ} \mathrm{F}$ or when a timeout period expired. The timeout period was used to trigger data acquisition even in the event that system didn't reach full temperature so that data were always acquired. At the end of the acquisition period LAMDA sent a signal to the helium system to start its shutdown sequence. DDS-Suite performed very well contributing to a successful test.

\section{F. CESTOL Test}

Although the processing and analysis portions of $D D S$-Suite were not used during the cruise-efficient short takeoff and Landing (CESTOL) test in the AEDC 40- by 80-Ft Wind Tunnel, LAMDA was used to acquire single microphone and phased microphone array data. For this test $L A M D A$ was modified to acquire video from an array mounted camera. The binary output files of $L A M D A$ were modified to work with the OptiNav array processing software used for the test. Data acquisition was triggered by the user because closed loop control was not allowed by the facility. $L A M D A$ performed flawlessly acquiring more than $1 \mathrm{~TB}$ of data.

\section{Summary}

At NASA Ames, requirements for a reliable, high-channel count, fast throughput dynamic-data system have been met with the development and deployment of DDS-Suite. Data acquisition, processing, and analysis of up to 672 channels of dynamic data with sample rates exceeding $200 \mathrm{kHz}$ with phase differences of less than $0.01^{\circ}$ has been demonstrated. Modularity and scalability make DDS-Suite useful for low or high channel-count tests in a variety of facilities. Automated acquisition and processing provide preliminary data in less than five minutes. QueryGUI is a user-friendly analysis tool to search and plot processed results in near real-time. Several large channel-count aeroacoustic test have been completed successfully using DDS-Suite. Near real-time data is critical for efficient, cost effective testing.

\section{References}

\footnotetext{
${ }^{1}$ Schreiner, J. A., Trosin, P. J., Pochel, C. A., and Koga, D. J., "DARWIN - Integrated Instrumentation and Intelligent Database Elements." AIAA Paper 96-2251, AIAA $19^{\text {th }}$ Advanced Measurement and Ground Testing Conference, New Orleans, Louisiana, June 17-19, 1996.

${ }^{2}$ Burnside, N.J., "IS-21 Acoustic Data Report.” NASA Internal Memo, October 17, 2006.

${ }^{3}$ Coe, Charlie, Personal interview, 2005.

${ }^{4}$ Burnside, N.J., "QueryGUI User Manual Release 1.78 CLV.” NASA Internal Memo, September 1, 2008.
} 